\title{
Fenología de la interacción girasol-Homoeosoma electellum Hulst. para el desarrollo de estrategias de control
}

\author{
Sunflower-Homoeosoma electellum Hulst. interaction phenology towards control \\ strategies development
}

\author{
Alán Rivero Aragón ${ }^{*}$, Horacio Grillo Ravelo ${ }^{2}$
}

\begin{abstract}
RESUMEN
El girasol es una planta anual, poco exigente de fertilizantes, de crecimiento rápido y extraordinaria resistencia a la sequía. Su principal plaga en gran parte de América es Homoeosoma electellum. Todos los daños ocasionados por esta plaga son producidos por su estado larvario, que solo permanece expuesto en la superficie de los capítulos por un corto período. El resto del ciclo larval se desarrolla protegido dentro de los aquenios o en el interior de los tejidos de tallos y capítulos, lo que hace el combate de esta especie extremadamente difícil. Se realizaron experimentos de campo evaluando varios cultivares de girasol, con el objetivo de establecer el momento ideal para realizar tratamientos contra Homoeosoma electellum. Se registró el momento de ocurrencia de los fenoestados de desarrollo críticos a partir de la emergencia. Estos momentos se asociaron a una escala de tiempo cronológico expresada en días y una escala de tiempo termal expresada en grados Celsius día de temperatura efectiva. Esto permite generalizar los resultados a regiones diversas de siembra. Se estableció que las aplicaciones de agentes de control deben hacerse durante el intervalo demarcado por 1.278 y 1.581 grados Celsius día. A partir de estos resultados es posible estimar los momentos críticos de infestación por Homoeosoma electellum para diferentes cultivares de girasol, con solo conocer cuándo ocurre su madurez fisiológica. De modo general el intervalo crítico para el combate de Homoeosoma electellum podría estimarse para cualquier cultivar de girasol a partir del $65 \%$ hasta el $84 \%$ de la duración del ciclo, desde la brotación hasta alcanzar la madurez fisiológica. Los momentos fenológicos de la interacción girasolHomoeosoma electellum establecidos permiten diseñar estrategias de combate efectivas y ecológicamente sostenibles.
\end{abstract}

Palabras clave: Helianthus annuиs, polilla del girasol, protección de plantas, control de plagas, tiempo termal

\begin{abstract}
Sunflower is an annual plant, not very demanding of fertilizers, fast growing and extraordinary resistance to drought. Its main pest in much of America is Homoeosoma electellum. This pest causes damage only during the larval stage. Larvae exposition on the surface of capitula is a quite short period. The rest of the larval cycle develops protected within the seeds, stems and capitula tissues. Hence control this species is extremely difficult. Field experiments on several sunflower cultivars were completed in order to establish a perfect timing to control Homoeosoma electellum. The time of occurrence of crucial phenological stages were registered from budding. These moments were adjusted to a thermal time scale expressed in Celsius degree day of effective temperature. This model allows applying the inferences to different harvest regions. Control agents applications must be carried out during the interval from 1,278 to 1,581 Celsius degree day. On this model might be possible to estimate the critic moments of Homoeosoma electellum infestation for others sunflower cultivars just knowing the moment of its physiological maturity. As a rule, a critical interval to control Homoeosoma electellum can be estimated between $65 \%$ and $84 \%$ of the cultivar cycle, from sprouting until reaching the physiological maturity. Establishing sunflower-Homoeosoma electellum interaction phenology allows designing effective and sustainable control strategies.

Keywords: crop protection, Helianthus annuus, pest control, sunflower moth, thermal time
\end{abstract}

\section{Introducción}

El girasol es uno de los cuatro cultivos de semillas oleaginosas más importantes en el mundo, y la calidad nutricional de su aceite comestible lo ubica entre los mejores aceites vegetales (Skoric et al., 2015). Es un cultivo oleaginoso de uso global, prometedor para la adaptación al cambio climático, ya que puede mantener rendimientos estables en una amplia variedad de condiciones ambientales, incluida

1 Departamento de Biología, Facultad de Ciencias Agropecuarias, Universidad Central Marta Abreu de Las Villas. Carretera a Camajuaní km 51/2, Santa Clara, Villa Clara, Cuba. E-mail: alanra@uclv.edu.cu

2 Centro de Investigaciones Agropecuarias, Universidad Central Marta Abreu de Las Villas

Fecha de recepción: 30 octubre, 2017.

Fecha de aceptación: 26 mayo, 2018.

DOI: 
la sequía (Badouin et al., 2017). Su principal plaga en gran parte de América es Homoeosoma electellum (Hulst) (Lepidoptera: Pyralidae). Todos los daños ocasionados por esta plaga son producidos por su estado larvario, que solo permanece expuesto en la superficie de los capítulos por un corto período de alrededor de seis días. El resto del ciclo larval se desarrolla protegido dentro de los aquenios o en el interior de los tejidos de tallos y capítulos. Esto hace el combate de esta especie extremadamente difícil después que las larvas han sobrepasado su segundo instar. Por otra parte, los primeros daños se hacen visibles cuando comienzan a acumularse en la superficie de los capítulos los desechos de la alimentación de las larvas y esto solo luego de una evaluación muy minuciosa. Las larvas que los produjeron se encuentran en instares superiores al tercero y por consiguiente ya protegidas en el interior de los tejidos de las plantas. Una infestación de cuatro larvas por planta incrementa los costos de producción y significa una pérdida de alrededor de 70 USD/hectárea como consecuencia del aceite que podría generarse. Todo esto hace que no sea recomendable esperar a que los daños sean visibles o confiar en un monitoreo de la abundancia de larvas para efectuar medidas de control, sino que lo más acertado es realizar tratamientos en el momento de máxima vulnerabilidad de las larvas. Este momento de máxima vulnerabilidad está asociado a los instares primero y segundo de su desarrollo, y estos a su vez, a la etapa durante la cual existen flores y polen para su alimentación, puesto que las larvas recién eclosionadas de H. electellum no son capaces de alimentarse de aquenios de girasol (Rivero-Aragón y Grillo-Ravelo, 2010a; b).

El presente trabajo se realizó con el objetivo de modelar los momentos fenológicos críticos de la interacción girasol-H. electellum para el desarrollo de estrategias de control eficaces y ecológicamente sustentables.

\section{Materiales y métodos}

Para establecer el momento de ocurrencia de los diferentes estados fenológicos de desarrollo, según la escala de Schneiter and Miller (1981), se realizaron tres experimentos de campo con cuatro cultivares de girasol, de características variables. Se asoció la ocurrencia de estos momentos a una escala de tiempo termal expresada en grados Celsius día $\left({ }^{\circ} \mathrm{Cd}\right) \mathrm{de}$ temperatura efectiva acumulada a partir del momento de emergencia.
Los cultivares probados fueron Caburé-15, CGGI-90, CGGI-611 (derivados de material genético de procedencia argentina) y CIAP JE94, seleccionado y estudiado en Cuba por su buena respuesta agronómica a partir de un material genético de nombre y origen desconocidos procedente de Chile (Alemán et al., 1999). Todos los experimentos se realizaron en condiciones agronómicas similares, solo que en diferentes momentos de siembra. Uno durante el mes de diciembre, la etapa en la que se sitúa el período óptimo para la siembra de girasol en Cuba y cuando aparecen menores afectaciones por $H$. electellum (Alemán, 2003); y dos sembrados fuera de la época óptima (septiembre y mayo, respectivamente). Producto de intensas lluvias durante la fase inicial del experimento de septiembre, las plantas no se desarrollaron bien, alcanzaron poca altura (entre 1,19 m y $0,53 \mathrm{~m}$ ) y resultaron delgadas para la variedad. No obstante, esto, lejos de perjudicar el cumplimiento del objetivo de los experimentos, produce mayor variabilidad a las condiciones de crecimiento y por consiguiente permite hacer inferencias más confiables, en condiciones diversas, acerca del desarrollo fenológico en función del tiempo termal. El área experimental se establece en la estación experimental Álvaro Barba, en el Centro de Cuba $\left(22^{\circ} 24^{1} \mathrm{~N}\right.$ y $\left.79^{\circ} 58^{\mathrm{l}} \mathrm{W}\right)$. Las densidades de siembra se mantuvieron alrededor de las 37.000 plantas/ha. No se realizaron aplicaciones de plaguicidas. Los registros de los estados fenológicos se hicieron semanalmente, evaluando 55 plantas por variedad cada vez.

La fecha de emergencia (cuando el $80 \%$ del total sembrado tiene los cotiledones por encima del suelo) se determinó por observación directa diaria. Los datos climáticos se obtuvieron de la estación agrometeorológica 78343 Yabú $\left(22^{\circ} 4^{\mathrm{I}} \mathrm{N}\right.$ y $\left.80^{\circ} 0^{\mathrm{I}} \mathrm{W}\right)$.

La suma térmica diaria (STd) se calculó según (Aiken, 2005):

$$
\mathrm{STd}=\frac{\text { TMaxd }+ \text { TMind }}{2}-\text { Cero biológico }
$$

Donde: TMaxd=Temperatura máxima diaria y TMind=Temperatura mínima diaria

Se considera como cero biológico para el girasol $4{ }^{\circ} \mathrm{C}$ (Aiken, 2005)

La suma térmica efectiva (ST) para un momento fenológico determinado se calcula como la sumatoria de STd acumulada hasta ese momento, a partir de la emergencia de las plantas (Aiken, 2005). Para calcular 
el filocrono medio, se determinaron las sumas térmicas promedio necesarias para que las plantas alcanzaran los consecutivos estados de desarrollo de la fase vegetativa del cultivo (Vn), los cuales son equivalentes al número de hojas en las plantas (ver Schneiter y Miller, 1981). Aplicando la ecuación:

$$
\mathrm{V}_{\mathrm{n}}=\mathrm{P}^{-1} * \mathrm{ST}
$$

Donde: $\mathrm{Vn}=$ fenoestado vegetativo $=$ número de hojas, $\mathrm{P}^{-1}=$ inverso del filocrono y ST = Suma térmica efectiva (Aiken, 2005)

A partir de la ecuación anterior, la suma térmica para la emisión de una hoja o filocrono $(\mathrm{P})$ puede ser calculada para cada fenoestado vegetativo como:

$$
\mathrm{P}=\frac{\mathrm{sT}}{\mathrm{V}_{\mathrm{n}}}
$$

Para estimar el filocrono para toda la etapa vegetativa en cada cultivar, se calcularon los valores promedio de filocrono para cada conjunto de datos. Las estimaciones de las medias se realizaron dentro de un 5\% de diferencia con la media real y un intervalo de confianza del $95 \%$. Para la comparación entre tratamientos se utilizaron análisis de varianza (ANOVA) seguidos de comparaciones múltiples de medias. Cuando no se pudo utilizar un análisis de varianza, por no cumplirse las asunciones necesarias para su adecuada interpretación, se emplearon pruebas no paramétricas que se indican en el caso.

\section{Resultados y discusión}

Con el resultado de las evaluaciones se creó una base de datos de alrededor de 6.000 observaciones de fenología. En el estudio se atienden, como objetivos de interés, dos fases fundamentales:. la primera se extiende desde la emergencia hasta que las flores comienzan a abrirse y aparece polen disponible, y la segunda desde este punto hasta que sucede la madurez fisiológica de los capítulos. Demarcadas de esta manera, la primera fase incluye todos los fenoestados vegetativos de la escala de Schneiter y Miller (1981), a partir de la emergencia y los primeros fenoestados reproductivos hasta que comienza la floración (R1 a R5 de la escala). La segunda fase de atención incluye todos los fenoestados durante los cuales ocurre la apertura progresiva de las flores (R5.1 a R5.9) y tiene su límite final en el fenoestado R6 de la escala mencionada. Generalmente, una buena medida para estimar la progresión fenológica de un cultivo, durante la etapa vegetativa, es la determinación de su filocrono (suma térmica (ST) necesaria para la aparición de una hoja). El concepto de filocrono se basa en la asunción de que la tasa de producción de nuevos fitómeros es cercana a una constante, desde la emergencia al inicio de la etapa reproductiva, cuando se expresa en unidades de tiempo termal (Chaves et al., 2017) (Della Noce et al., 2016). Aiken (2005) sostuvo el concepto de filocrono para girasol y lo estableció en 37,3 $\pm 1,3$ grados Celsius día $\left({ }^{\circ} \mathrm{Cd}\right)$ hoja $^{-1}$ en cultivo de secano y $36,1 \pm 1,4$ con suficiencia de agua, según el método lineal de cálculo de suma térmica. Como resultado del presente estudio se obtuvo una suma térmica promedio para la emisión de una hoja, considerando el conjunto de los cultivares probados, de $43,5^{\circ} \mathrm{Cd} \mathrm{hoja}^{-1}$ (intervalo de confianza 95\%: 38,2 - 48,7 ${ }^{\circ} \mathrm{Cd} \mathrm{hoja}^{-1}$ ).

No obstante, a partir de las observaciones realizadas se pudo determinar que los valores de suma térmica, necesarios para la emisión de una hoja, tienen una tendencia logarítmica descendente con el incremento del número de hojas emitidas (Figura 1). De esta forma las plantas necesitan una cantidad cada vez menor de grados efectivos de calor acumulados, para emitir la próxima hoja, según se van desarrollando. Este resultado se opone al establecimiento de filocrono en girasol como una constante durante la etapa vegetativa. Ya Villalobos y Ritchie (1992) habían previsto un filocrono variable al establecer $38,7{ }^{\circ} \mathrm{Cd}$ hoja $^{-1}$ y $23^{\circ} \mathrm{Cd} \mathrm{hoja}^{-1}$ antes y después del fenoestado V6, respectivamente. La divergencia con los resultados de Aiken (2005), quien establece valores constantes de suma térmica para emitir una hoja, se debe a que los resultados proporcionados en su estudio surgen de la asunción de una relación lineal entre la aparición de hojas (hasta V14) y la suma térmica acumulada a partir de la emergencia.

Aunque se desestime la utilización de un filocrono como constante para predecir momentos de ocurrencia de estados fenológicos, los valores promedio de suma térmica para emitir una hoja ofrecen información acerca de la precocidad de los cultivares probados, así como de las diferencias para el desarrollo de las plantas de girasol en relación con estudios realizados en otras latitudes.

Según estos estudios, el cultivar CIAP JE 94 necesita significativamente menor suma térmica promedio para la emisión de hojas que el resto de los cultivares, lo que es un buen indicador de precocidad para alcanzar fenoestados reproductivos (Figura 2).

Al comparar los valores de suma térmica y edad que necesitaron los diferentes cultivares para 


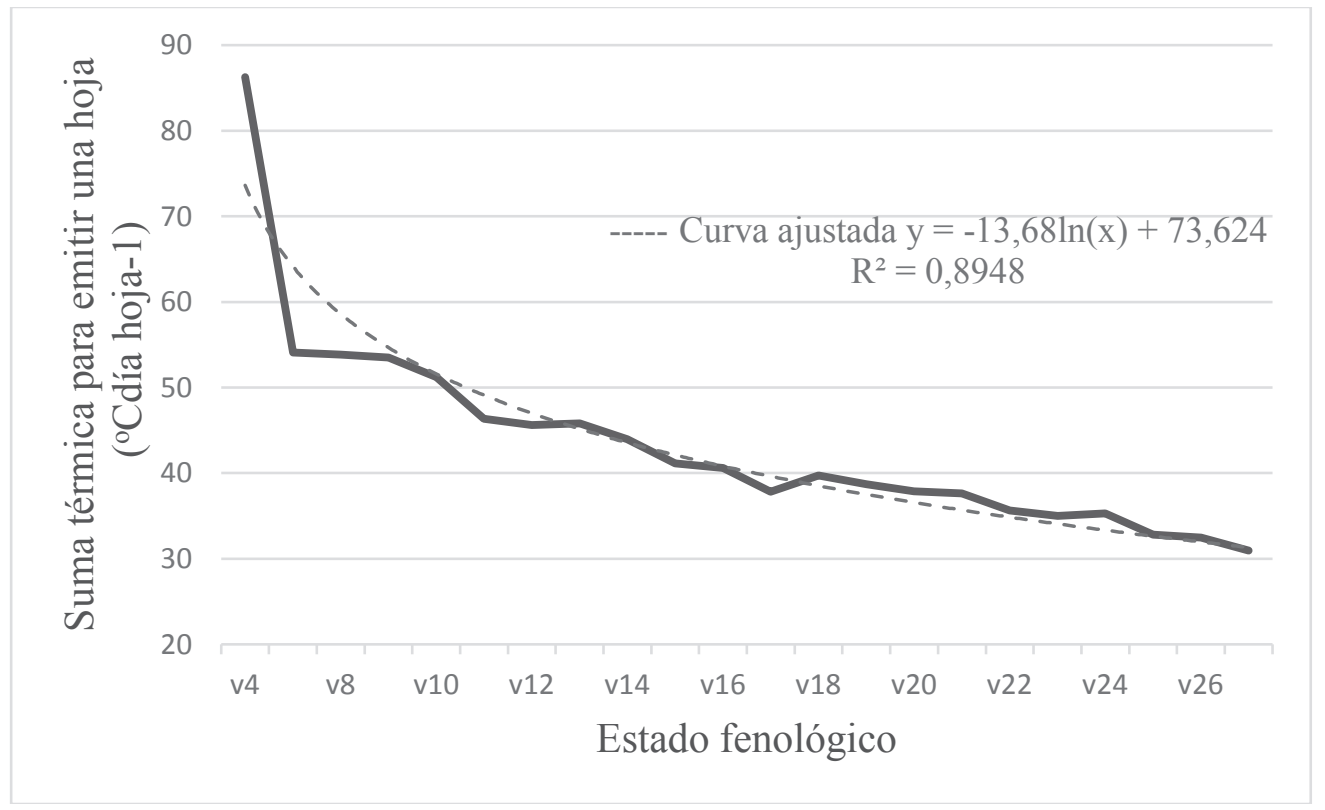

Figura 1. Suma térmica promedio para la emisión de una hoja por estado fenológico.

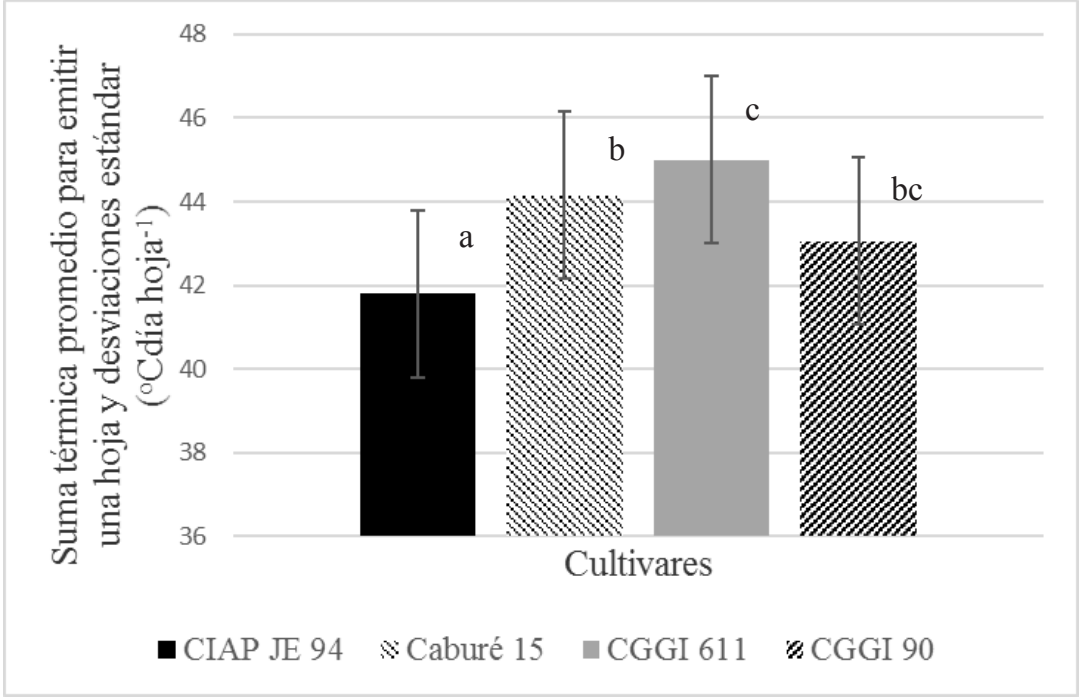

Figura 2. Suma térmica promedio para la emisión de una hoja, por cultivar de girasol. Letras no coincidentes indican diferencias estadísticas significativas (ANOVA por rangos de Friedman y prueba de Wilcoxon para datos pareados con corrección de Bonferroni, $\mathrm{p}<0,01$ ) 
desarrollarse hasta los fenoestados R4 (inicio de apertura de la inflorescencia) y R6 (madurez fisiológica), se pueden establecer dos grupos de variedades (ver Tablas 1 y 2). El primer grupo, más precoz, está integrado por las variedades CIAP JE 94, Caburé 15 y CGGI 611. Necesita menos de $70 \mathrm{~d}$ y $1.300^{\circ} \mathrm{Cd}$ para desarrollarse hasta el fenoestado R4 y menos de $80 \mathrm{~d}$ y $1.600^{\circ} \mathrm{Cd}$ para desarrollarse hasta el fenoestado R6. El segundo grupo está conformado por la variedad CGGI 90, que necesita como promedio $70 \mathrm{~d}$ y $1.391{ }^{\circ} \mathrm{Cd}$ para alcanzar el fenoestado $\mathrm{R} 4$ y $83 \mathrm{~d}$ y $1.618^{\circ} \mathrm{Cd}$ para llegar al fenoestado R6.

Tabla 1. Suma térmica necesaria para alcanzar el fenoestado $\mathrm{R} 4$, por cultivares. $\left({ }^{\circ} \mathrm{Cd}\right)$

\begin{tabular}{lccc}
\hline \multicolumn{1}{c}{ Cultivar } & Media & Error estándar & Intervalo confianza 95\% \\
\hline CIAP JE 94 & $1.260,9 \mathrm{a}$ & 14,0 & $1.233,3-1.288,5$ \\
Caburé 15 & $1.268,0 \mathrm{a}$ & 16,6 & $1.235,2-1.300,8$ \\
CGGI 611 & $1.274,5 \mathrm{a}$ & 17,3 & $1.240,3-1.308,7$ \\
CGGI 90 & $1.390,7 \mathrm{~b}$ & 15,0 & $1.361,2-1.420,3$ \\
\hline
\end{tabular}

Letras no coincidentes indican diferencias estadísticas significativas (ANOVA y prueba de Newman-Keuls para comparación múltiple de medias, $\mathrm{p}<0.000$ )

Tabla 2. Suma térmica necesaria para alcanzar el fenoestado $\mathrm{R} 6$ por cultivares. $\left({ }^{\circ} \mathrm{Cd}\right)$

\begin{tabular}{|c|c|c|c|}
\hline Cultivar & Media & Error estándar & Intervalo confianza 95\% \\
\hline CIAP JE 94 & $1.522,9 \mathrm{ab}$ & 13,3 & $1.496,8-1.549,0$ \\
\hline Caburé 15 & $1.547,3 \mathrm{a}$ & 11,4 & 9,8 \\
\hline CGG & $1.569,4 \mathrm{~b}$ & 12 & 6 \\
\hline CGG & $1.617,6$ & 12,8 & 1.5 \\
\hline \multicolumn{4}{|c|}{$\begin{array}{l}\text { Letras no coincidentes indican diferencias estadísticas significativas } \\
\text { (ANOVA y prueba de Newman-Keuls para comparación múltiple } \\
\text { de medias) } p=0,02 \text { para Caburé } 15 \text { contra CGGI } 611 \text {; para el resto } \\
\mathrm{p}<0,01\end{array}$} \\
\hline
\end{tabular}

Una vez que las plantas de girasol superan la etapa de su fenología en que hay flores y polen disponible en los capítulos, las larvas recién emergidas de H. electellum no pueden alimentarse y por consiguiente desarrollarse, aun cuando continúe la actividad reproductiva de los adultos (Rivero-Aragón y Grillo-Ravelo, 2010a). Teniendo en cuenta esta conducta, se define el periodo crítico para evitar las infestaciones entre la aparición de las primeras flores y la madurez fisiológica. Para fijar el momento en el cual se inician las infestaciones por H. electellum al girasol en cada grupo de variedades, se utilizaron los límites inferiores al 99\% de confianza, de las medias de suma térmica a las cuales ocurre el fenoestado R4. A su vez, como indicadores del momento crítico en el cual terminan las oportunidades de infestación, se emplearon los límites superiores también al 99\%, de las medias de suma térmica para las cuales ocurre el fenoestado R6 (Tabla 3). Los tamaños de muestra fueron suficientes para hacer estos estimados con menos de $10 \%$ de diferencia con la media real.
Tabla 3. Suma térmica que marca los momentos críticos para el inicio y terminación de la infestación de H. electellum en girasol $\left({ }^{\circ} \mathrm{Cd}\right)$

\begin{tabular}{lccc}
\hline \multicolumn{1}{c}{ Cultivar } & Inicio & Final & Diferencia \\
\hline Todos los cultivares & 1.278 & 1.581 & 303 \\
Cultivares CIAP JE 94, Caburé & 1.244 & 1.566 & 322 \\
15 y CGGI 611 & 1.346 & 1.649 & 303 \\
Cultivar CGGI 90 & &
\end{tabular}

Si se realizan aplicaciones de compuestos activos o medios biológicos para el combate de $H$. electellum, durante el intervalo demarcado por estos límites, se pueden proteger las plantaciones mientras dura el período de floración para la mayoría de las plantas (R5 hasta R6) y se extiende además esta protección a plantas que florecen tempranamente, cuando la mayor parte se encuentra en el fenoestado anterior (R4). Estas plantas precoces pueden generar infestaciones a partir de larvas en instares superiores al tercero (L3). Las larvas en estados superiores a L3 son ya capaces de alimentarse de la base de los capítulos, de los tallos y aquenios formados. No necesitan la presencia de polen para iniciar la infestación en una planta y pueden pasarse de unas a otras. Estas larvas producto de infestaciones tempranas son, por otra parte, muy difíciles de combatir, puesto que se encuentran protegidas dentro de los tejidos de las plantas cuando el resto de las larvas están en su etapa más vulnerable.

De los resultados se puede establecer para todos los cultivares que, como tendencia central, el fenoestado R4 ocurre al concluir una fracción alrededor de 0,7 del total del ciclo hasta la madurez fisiológica, marcada por el fenoestado R9 (intervalo de confianza 95\%: estimado tiempo termal 0,65-0,71) (Figura 3). Y el fenoestado R6 en una e fracción de 0,8 de este mismo total (intervalo de confianza 95\%: estimado con suma térmica 0,80-0,84).

Este modelo se puede aplicar a otros cultivares de girasol, dentro de los límites de certeza que ofrece este trabajo, a partir de saber cuándo ocurre su madurez fisiológica. De esta manera el intervalo crítico para el combate de H. electellum puede estimarse entre el $65 \%$ de la duración del ciclo hasta alcanzar el fenoestado R9 y el 84\% de la duración del ciclo hasta alcanzar el mismo fenoestado.

Los momentos fenológicos de la interacción girasolHomoeosoma electellum establecidos permiten diseñar estrategias de combate efectivas y ecológicamente sostenibles. De acuerdo a este modelo, las medidas de combate aplicadas estarían concentradas en un corto periodo, reduciendo los costos e incrementando la eficacia. Sería además aconsejable la utilización de 


\begin{tabular}{lr:rr} 
Cultivares CIAP JE 94, Caburé 15 y CGGI 611 & $1267^{\circ} \mathrm{Cd}$ & $1548^{\circ} \mathrm{Cd}$ & $1881^{\circ} \mathrm{Cd}$ \\
\hline Cultivar CGGI 90 & $64 d$ & $77 d$ & $97 d$ \\
\hline Estado fenológico & $1391{ }^{\circ} \mathrm{Cd}$ & $1618^{\circ} \mathrm{Cd}$ & $1961^{\circ} \mathrm{Cd}$ \\
\hline Porcentaje del desarrollo hasta R9 & $70 d$ & $83 d$ & $103 d$ \\
\hline Intervalo crítico para el combate de H. electellum & $65 \%$ & $R 6$ & $R 9$ \\
\hline
\end{tabular}

Figura 3. Momentos fenológicos críticos para el combate de H. electellum expresados como porciento del desarrollo, desde la emergencia hasta el fenoestado R9.

medios biológicos para acompañar este modelo, como alternativa de agente de control.

\section{Conclusiones}

El período crítico para proteger al girasol de infestaciones por $H$. electellum está comprendido en el intervalo de 1.278 a $1.581{ }^{\circ} \mathrm{Cd}$ de temperatura efectiva. Para todos los cultivares probados, el fenoestado R4 ocurre como promedio al acumularse un $70 \%$ del total de temperatura efectiva necesaria para alcanzar la madurez fisiológica del cultivo desde la emergencia y el fenoestado R6 en un $80 \%$ de este mismo total. Un intervalo crítico seguro para proteger al girasol de infestaciones por $H$. electellum, puede estimarse entre el 65\% de la duración del ciclo de los cultivares hasta alcanzar el fenoestado R9 y el 84\% de la duración del ciclo hasta alcanzar el mismo fenoestado.

\section{Literatura citada}

Aiken, R.M.

2005. Applying Thermal Time Scales to Sunflower Development. Agron. J., 97 (3): 746.

Alemán, R.

2003. El cultivo del girasol ( Helianthus annuus L .) en el marco de una agricultura sostenible. Rev. Cent. Agrícola, 30 (1): 90-91.

Alemán, R., D. Martín, C. Hernández, y E. Méndez.

1999. Caracterización y extensión de la variedad de girasol CIAP JE -94. Rev. Cent. Agrícola, 26 (2): 25-27.

Badouin, H., J. Gouzy, C.J. Grassa, F. Murat, S.E. Staton, L. Cottret, C. Lelandais-Brière, G.L. Owens, S. Carrère, B. Mayjonade, L. Legrand, N. Gill, N.C. Kane, J.E. Bowers, S. Hubner, A. Bellec, A. Bérard, H. Bergès, N. Blanchet, M.-C. Boniface, D. Brunel, O. Catrice, N. Chaidir, C. Claudel, C. Donnadieu, T. Faraut, G. Fievet, N. Helmstetter, M. King, S.J. Knapp, Z. Lai, M.-C. Le Paslier, Y. Lippi, L. Lorenzon, J.R. Mandel, G. Marage, G. Marchand, E. Marquand, E. Bret-Mestries, E. Morien, S. Nambeesan, T. Nguyen, P. Pegot-Espagnet, N. Pouilly, F. Raftis, E. Sallet, T. Schiex, J. Thomas, C. Vandecasteele, D. Varès, F. Vear, S. Vautrin, M. Crespi, B. Mangin, J.M. Burke, J. Salse, S. Muños, P. Vincourt, L.H. Rieseberg, and N.B. Langlade.

2017. The sunflower genome provides insights into oil metabolism, flowering and Asterid evolution. Nature, 546 (7656): 148-152. Chaves, G.G., A. Cargnelutti Filho, B.M. Alves, A. Lavezo, C.A. Wartha, D.B. Uliana, R.V. Pezzini, J.A. Kleinpaul, I.M.M. Neu, G.G. Chaves, A. Cargnelutti Filho, B.M. Alves, A. Lavezo, C.A. Wartha, D.B. Uliana, R.V. Pezzini, J.A. Kleinpaul, and I.M.M. Neu. 2017. Phyllochron and leaf appearance rate in oat. Bragantia, 76 (1): 73-81.
Della Noce, A., V. Letort, A. Hansart, C. Baey, G. Viaud, S. Barot, J.-C. Lata, X. Raynaud, P.-H. Cournede, and J. Gignoux. 2016. Modeling the inter-individual variability of singlestemmed plant development. In 2016 IEEE International Conference on Functional-Structural Plant Growth Modeling, Simulation, Visualization and Applications (FSPMA). IEEE. pp. 44-51.

Rivero-Aragón, A., y H. Grillo-Ravelo.

2010a. Observaciones sobre la conducta alimentaria y reproductiva de Homoeosoma electellum ( Hulst .) en Cuba. Cent. Agrícola, 37 (3): 83-87.

Rivero-Aragón, A., y H. Grillo-Ravelo.

2010b. Evaluación de los daños producidos por Homoeosoma electellum (Hulst) a girasol en Cuba Evaluation of the damages produced by Homoeosoma electellum (Hulst) to sunflower in Cuba. Cent. Agrícola, 37 (4): 9-14.

Schneiter, A.A., and J.F. Miller.

1981. Description of Sunflower Growth Stages1. Crop Sci., 21 (6): 901.

Skoric, D., Z. Sakac, and Y. Demurin.

2015. Genetic Variability of Oil Quality Components in Sunflower As a Function of Developing Hybrids with Novel Oil. J. ASM. Life Sci., 326 (2): 138-146.

Villalobos, F.J., and J.T. Ritchie.

1992. The effect of temperature on leaf emergence rates of sunflower genotypes. F. Crop. Res., 29 (1): 37-46. 